\title{
A Review of Inconsistencies and Unfounded Assumptions in Physics Enables A Path Forward
}

\author{
William Oakley* \\ Retired Scientist, Valerio St. Santa Barbara, California, USA
}

\section{Article Info}

*Corresponding author:
William Oakley
Retired Scientist
Valerio St. Santa Barbara, California
USA
Tel: +8057709811
E-mail: oakleyw137@gmail.com

Received: November 16, 2020 Accepted: December 07, 2020

Published: December 18, 2020

Citation: Oakley W. A Review of Inconsistencies and Unfounded Assumptions in Physics Enables a Path Forward. Int J Cosmol Astron Astrophys. 2020; 2(1): 115-120.

doi: 10.18689/ijcaa-1000124

Copyright: (c) 2020 The Author(s). This work is licensed under a Creative Commons Attribution 4.0 International License, which permits unrestricted use, distribution, and reproduction in any medium, provided the original work is properly cited.

Published by Madridge Publishers

\begin{abstract}
It is widely recognized significant parts of leading-edge physics are at an impasse. Perhaps it is time to re-evaluate long-standing inconsistencies and assumptions that have become dogma but are erroneous and blocking progress. Newton's gravitational constant $G_{N}$ is assumed a natural constant, having originated via Newton's notion of gravity as radial force acting on mass in flat observer space. But Einstein showed gravity due to curved space time with "mass" dimensionally c ${ }^{2}$ remote from the observer energy domain. Dirac stated (elementary) particles are "no more than electromagnetic energy localized in observer space". This suggests gravity is emergent at the particle scale by spacetime curved in three dimensions. But Newton's assumed radial force is consistent only with spacetime curvature in the two dimensions orthogonal to the radial, so how can $G_{N}$ be fundamental? Do the different dimensionalities of Newton's and Einstein's theories relate to the Dark Matter issue?
\end{abstract}

Describing the electron as a photon in a relativistic quantum loop localized by curved spacetime enables derivation of an expression for $G_{N}$ giving a value within the empirical uncertainty. The electron is posited as relativistic electromagnetic energy in dynamic equilibrium between circumferential metric tension at the Strong Force scale and radial electrostatic force, satisfying the Planck "Force Equality" premise. As historically long suspected $G_{N}$ contains a numerical factor of $c^{4}$, derived from the cgs units, in which it was first measured, and a relativistic factor, $\alpha^{-4 / 3}$, which move the Planck scale into exact correspondence with the electron parameters. General Relativity is shown a fundamental femto-scale theory where the strong force in a metric curved at the particle scale is manifest in observer space reduced by the classical "Large Number" of $5.7 \times 10^{44}$ and is evident as gravity.

The expression obtained for $G_{N}$ is supported by deriving the MOND constant and the observed flat galactic star rotation velocity curves.

Resolving identified erroneous assumptions and inconsistencies will significantly impact cosmology and particle physics and bring gravitational and electromagnetic unification closer.

Keywords: Electron; Dark Matter; Planck Mass; Newton's G; Large Numbers; Quantum gravity; Proton mass anomaly; MOND.

\section{Introduction}

Progress in fundamental physics has slowed substantially in recent decades with new discoveries seeming increasingly minor and peripheral to fundamental issues. High energy physics is well funded and vigorously pursued, but shows little recent progress while a significant number of basic issues are routinely ignored, (e.g. how does the 
electron exhibit gravity? Why is the proton rest mass so much greater than the electron's? ). Why does physics drive to find more high energy "resonances" that do not address existing basic issues? Did finding the Higgs boson help to understand and compute the rest masses of stable particles, or extend the Standard Model to a new family of more massive particles? Or address the Large Number issue? No. Physics is facing a plethora of unresolved basic questions. Perhaps the lack of real progress stems from entrenched erroneous assumptions and ongoing failure to address and resolve conflicting theories.

Newton's empirical constant $G_{N^{\prime}}$ arose with his 1687 gravitational equation [1], now outdated and long superseded by Einstein's 1917 General Theory of Relativity, (GR) [2]. But $G R$ is incomplete as it lacks a connection to both $G_{N}$ and the atomic scale. Consequently, $G_{N}$ remains useful but is theoretically unsupported so why is it still believed a fundamental constant? The Planck scale of matter [3] is conjectured to exist at a mass energy about 20 orders of magnitude greater than the elementary particles, but this factor depends on the value of $G_{N^{\prime}}$ an isolated and empirical construct, so it is also theoretically unsupported at a fundamental level. Cold Dark Matter, (CDM), is assumed to exist although decades of searching have produced no positive results, only myriad speculative articles, and it likely does not exist [4]. Does the CDM problem arise from using Newton's radial force equation to calculate masses of orbital celestial bodies whereas Einstein's General Relativity (GR), ascribes gravitational orbits to curved spacetime? GR has survived all tests and is now considered proven but is not connected to either $G_{N}$ or particle physics. Consequently, as gravity originates at the particle scale GR must be incomplete. Logic dictates observer space gravity is a manifestation of forces acting at the particle scale which, per Dirac, localize electromagnetic (EM) energy and enable particle formation, e.g. the electron. This should be the focus of our attention, not the Large Hadron Collider (LHC), String Theory, Multiverse conjectures or similar efforts that do not address these and other basic issues, and which many still deem "intractable".

Physicists are aware of these issues, but the standard community approach of incremental progress via peer reviewed journal articles is not well-suited to identify and correct outdated and /or contradictory notions and erroneous dogma. These problems are difficult, if not impossible to address in a strongly competitive peer pressure "publish or perish" environment, which has led physics into a speculative morass [5] ("No problem can be solved from the same level of consciousness that created it."-Albert Einstein).

\section{The Planck Scale relation assumes Newton's $\mathrm{G}_{\mathrm{N}}$ Fundamental, But Is It?}

The pace of discovery in High Energy Particle (HEP) physics has slowed to a crawl and all but stopped. This likely presages the end for HEP but not perhaps for particle physics in general. The LHC has produced essentially no results in its search for another generation of particles to fill the large gap, (the Hierarchy problem) [6], between those now known, e.g. the Higgs Boson [7] at $125 \mathrm{GeV}^{\text {a }}$ and the (conjectured) Planck Scale at $1.2 \times 10^{19} \mathrm{GeV}$. Perhaps no particles exist in this huge range, or perhaps the Planck scale is in error and the Higgs boson is essentially the end of the line. Is that plausible? The presently assumed Planck mass scale is defined as where the fundamental forces are equal, (gravity, Electromagnetic, Strong Force), with the Planck Mass $M_{p}$ obtained by equating Newton's gravitational expression $F_{g}=G_{N} M m / r^{2}$, [1] and the Strong force $F s=\hbar c / r^{2}$, i.e. $\hbar c / r^{2}=G_{N} M_{p}^{g} / r^{2}$, giving

$$
M_{p}=\left(\hbar c / G_{N}\right)^{1 / 2}
$$

But a presumed (Newtonian) gravitational force in the observer domain and the Strong force within subatomic particles are in different spacetimes, so how can equating them be meaningful? The Planck scale is clearly problematic. However, the notion of force equality must be valid for elementary particle stability in femto-scale domains where observer gravity and $G_{N}$ are essentially irrelevant.

It may be heresy but the answer to some outstanding issues will not be found at the high energy extreme but at the low energy scale of the electron. The electrostatic and strong forces are equal within the electron (classically $e^{2} / r^{2}=\alpha \hbar c / r^{2}$ ), which suggests a particle in dynamic equilibrium and rotationally relativistic by $\alpha$, where $\alpha^{-1} e^{2} / r^{2}=\hbar c / r^{2}$, and with the charge energy apparent to the observer reduced by the relativistic rotation factor $\alpha$ (the fine structure constant). Is it plausible this equality exists over the huge energy range from the electron to the Planck scale? No, so if the Planck scale is in error the problem lies with $G_{N^{\prime}}$ the least certain of the Planck relation parameters in eq. (1).

\section{The Origin of Newton's G}

$G_{N}$ arose with Newton's gravity equation describing a radial gravitational force in the observer domain between two observer domain masses. It has long been replaced by Einstein's General Relativity (GR), wherein gravity is described as acting via curved spacetime. GR is a macro scale theory and does not explain how gravity emerges at the particle scale, and so does not contain a scaling factor that provides a value for $G_{N}$. With no accepted way to quantify gravity via $G R$, Newton's $G_{N}$ remains part of empirical physics but is isolated and unsupported by theory. It is an outlying parameter unrelated to the atomic constants and in this context is suspect \#1. GR shows the observer domain radial force described by Newton's gravity equation does not exist. But if GR is essentially correct how can $G_{N}$ be a natural constant as dogma insists? It is a prime candidate for revision.

\footnotetext{
a Standard abbreviations are used for all quantities (e.g. GeV, $M e V, c g s, G_{N}$, and constants, (e.g. $m_{e^{\prime}} m_{p^{\prime}} c, \alpha$, $\hbar$, etc.) ${ }^{b}$ This is no longer believed true, See reference [8].
} 


\section{The large number problem}

Dirac introduced his Large Number Hypothesis (LNH), in 1937 relating the scale of the Universe to the mass of the proton, obtaining a nominal factor of $10^{39}$ [8-10]. The hypothesis retained $G_{N}$ as a natural parameter but implied it was not constant and changed value with the age of the universe. Another classical large number is given by the ratio Strong Force $\left(\hbar c / r^{2}\right)$, to classical gravity for the electron, $\mathrm{G}_{\mathrm{N}} \mathrm{m}_{\mathrm{e}}^{2} / \mathrm{r}^{2}$, evidencing a Large Number $L N$, unrelated to the universe age [11].

$$
L N=\hbar c / G_{N} m_{e}^{2}=5.7084 \times 10^{44}
$$

In contrast to Dirac's $L N H$, as shown below, $G_{N}$ is neither natural nor a Universal constant and its value, and $L N$, are highly dependent on the light velocity value $c$, in the units in which $G_{N}$ was first measured.

A value for Newton's $G_{N}$ was first obtained by Cavendish in 1798 when cgsi units were in vogue (with light velocity $c=2.9979 \times 10^{10} \mathrm{~cm} / \mathrm{sec}$ ), and it has often been noted the fourth power of the numerical value of $c$ in those units, (ㄷ say), is very large, with $\underline{c}^{4}=8.0773 \times 10^{41}$. This number differs from LN by a relatively small factor, about 706.7. As the Large Number expression (2) contains a value for $G_{N}$ it is likewise theoretically unsupported at a basic level.

The electron is described [11] as a photon of free space energy $\hbar c / r$, wrapped $\alpha^{-1}$ times around the particle $z$ axis to form a spacetime curvature of mass energy $\alpha^{-1} m_{e} c^{2}$, and adopts a relativistic state where a volume expansion by $\alpha^{-1},(\approx$ $137)$, reduces the circumferential propagating energy by $\alpha^{1 / 3}$ to $\alpha^{-2 / 3} m_{e} c^{2}$. The expansion also reduces the radial charge energy in the observer domain by $\alpha$ to $\alpha \hbar c / r=e^{2} / r$ with the apparent radial mass reduced to $\mathrm{m}_{\mathrm{e}}$. The total energy is thereby preserved at zero, $m_{e} c^{2}-\alpha \hbar c / r=0$. Particle stability in the relativistic rotating domain indicates $\alpha^{-1} m_{e} c^{2}-\hbar c / r=0$, with the mass energy distributed in three dimensions. A radial toward the observer is normal to a circumferential strong force in spacetime curved in $2 \mathrm{D}$ and the circulating mass energy is thereby evident to the observer decreased by $\alpha^{-2 / 3} c^{2}$, appearing as a radial mass effect. The implied gravitational attraction between two electrons thus differs only slightly from the classical Large Number in eq (2) and weaker than the strong force by

$$
\left(\alpha^{-2 / 3} \underline{c}^{2}\right)^{2}=5.7071 \times 10^{44}
$$

Dirac stated [8], (circa 1928), that "all matter is no more than localized electromagnetic (EM) energy", now long since proven by low energy electron-positron annihilation which produces only EM photons, but EM energy only propagates rectilinearly at light speed in vacuo. So logically the electron must be EM energy localized by propagating rectilinearly in spacetime highly curved at the particle scale. And exhibiting mass due to propagation at a relativistic velocity close to $\mathrm{c}$. Newton's equation contains a gravitating mass term $\mathrm{m}^{2}$, thus if $\mathrm{m}^{2}=706.7$ as above, $\mathrm{m}=26.58$, and the circulating relativistic mass energy in each electron of rest mass $m_{e}$ is $m \approx 26.58$ $m_{e} c^{2}$, with each spin half component $13.58 m_{e} c^{2}$ at $6.791 \mathrm{MeV}$.
This is posited as a gravity quantum, $\left(\mathrm{m}_{\mathrm{q}} \mathrm{c}^{2} \approx 6.791 \mathrm{MeV}\right)$; which in a quantum loop relativistic by $\alpha^{-1 / 3}$ gives a loop energy of $35.01 \mathrm{MeV}$. Two spin- $1 / 2$ electron loops each of 35.01 MeV form a circulating spin-1 photon of $70.02 \mathrm{MeV}$, evident to the observer reduced by $\alpha$ to $0.511 \mathrm{MeV}$, the electron rest mass energy.

\section{The Planck scale}

The notional Planck Mass scale [3], was introduced circa 1895. Updating the Planck mass value given in eq (1) where $\mathrm{G}=\hbar c / \mathrm{M}_{\mathrm{p}}^{2}$ with

$$
\mathrm{G} \approx \hbar c / \mathrm{m}_{\mathrm{e}}{ }^{2}\left(\alpha^{-2 / 3} \underline{c}^{2}\right)^{2}
$$

shows the "Planck Mass" an energy $\alpha^{-2 / 3} \mathrm{~m}_{\mathrm{e}} \mathrm{c}^{2} \approx 13.582$ $\mathrm{MeV}$, the relativistic photon energy propagating circumferentially within the electron. Consequently, there is no 'large gap' in particle energies and the 'hierarchy problem' no longer exists. The Planck force equality criteria is satisfied within the electron as observer domain gravity is just a manifestation of the circumferential Strong Force at the particle scale, and GR, Newton's $G_{N^{\prime}}$ and electrodynamics are connected via the nominal scaling factor $\left(\alpha^{-2 / 3} \underline{c}^{2}\right)^{2}$.

Due to not understanding the true nature of gravity, Newton's $G_{N}$ although useful, is neither fundamental nor natural. The gravitational effect between two electrons arises from the circumferential strong force reduced by a scaling factor $\left(\alpha^{-2 / 3} \underline{c}^{2}\right)^{2}$, essentially the Large Number, providing a direct connection between the curved spacetime of GR (at the femto-scale), and the EM photon circulating within the electron.

Relation (4), $\mathrm{G} \approx \hbar c / \mathrm{m}_{\mathrm{e}}^{2}\left(\alpha^{-2 / 3} \underline{c}^{2}\right)^{2}$, gives a numerical value for $G$ just outside the empirical CODATA uncertainty of $G_{N}$. If the energies circulating in the electron's two quantum loops cross couple, a small mass adjustment factorii of 1.00013725 is obtained, giving the empirical value of LN, and a gravity quantum,

$$
\mathrm{m}_{\mathrm{q}} \mathrm{c}^{2}=6.792131227 \mathrm{MeV}
$$

and a slightly revised expression for $G_{N^{\prime}}$

$$
G_{N}=\hbar c /\left(2 m_{q} \underline{c}^{2}\right)^{2}
$$

giving a numerical value within the CODATA empirical standard uncertainty at $G_{N}=6.67384 . \pm 0.00080$.

$$
\mathrm{G}_{\mathrm{N}}=6.674273033 \times 10^{-11} \mathrm{SI} \text { Units }
$$

\section{Does Dark Matter Exist?}

Cosmological observations seem to show an unidentified source of mass pervades the Universe as the motion of stars orbiting in the outer regions of galaxies do not appear to follow Newtonian dynamics with the mass of orbiting matter too low by a factor of about 5.2 as computed from other observational data; i.e. the measured radii and velocities of stars orbiting in outer regions of galaxies, with expected masses estimated by observed star parameters. The notion of Cold Dark Matter (CDM) was invented to solve this "low 
observed mass" problem and the search for it has been ongoing for some time, almost a century, with absolutely no trace to be found, out in deep space, in deep underground mines, nor anywhere else. The lack of any physical evidence for a "dark matter" particle has caused some to wonder if it really exists [12], and if it does not what is the problem? An attempt to resolve the problem was made with the introduction of MOND theory, (Modified Newtonian Dynamics), but was not successful. The search continues (Charlie Wood, Quanta Magazine Nov. 27. 2019), but it is becoming clear the solution to the CDM issue will probably not involve a new particle.

The electron is described above as a single spin- 1 photon localized in space as two orthogonal relativistic spin-1/2 quantum loops with the particle relativistic volume expanded by $1 / \alpha$ and each loop two dimensional and expanded by $\alpha^{-2 / 3}$. The total particle mass energy in three dimensions is $\alpha^{-1} \mathrm{~m}_{\mathrm{e}} \mathrm{c}^{2}$ but the circumferential mass energy via which Newton's $G_{N}$ is derived is $\alpha^{-1 / 3}$ less at $\alpha^{-2 / 3} \mathrm{me}_{\mathrm{e}} \mathrm{c}^{2}$. The circulating relativistic energy is orthogonal to all particle radials and evident to the observer reduced by $\alpha^{-2 / 3} c^{2}$ to $m_{e}$. A similar factor applies to nucleons, and the mass of objects measured along a radial will thereby evidence a mass lower by $\alpha^{-1 / 3}$, about 5.1560, compared to a total mass value derived via non-radial measurement. In contrast to Newtonian dynamics, General Relativity (GR) describes masses in gravitational orbits as propagating rectilinearly in curved spacetime, and not radially attracted. For objects in gravitational orbits this implies a total mass greater by $\alpha^{-1 / 3}$ than deduced using Newtonian dynamics, i.e. the observer perceives objects in unaccelerated gravitational orbits propagating as though centrally attracted by an effect of magnitude $G M \alpha^{-1 / 3} \mathrm{~m} / \mathrm{R}^{2}$, which does not exist.

It is concluded CDM does not exist and the problem arises from using Newton's gravitational expression to calculate the mass of objects orbiting galaxies by propagating rectilinearly in curved spacetime.

Current values for the Universe composition give normal baryonic matter at $4.6 \%$, Cold Dark Matter (CDM), at 24\%, and Dark Energy (DE), at $71.4 \%$. But if CDM does not exist and normal matter is low by 5.156 , then the normal matter should be $23.72 \%$. However, $23.7 \%$ and $71.4 \%$ only add to $95.1 \%$, so both normal matter and DE values should be increased by $1 / 0.951=1.0515$, giving $D E=75.08 \%$ and Normal baryonic matter $=24.92 \%$.

\section{The Proton Anomaly}

How does the Proton mass energy at $938.272 \mathrm{MeV}$ relate to the previously described electron mass energy configuration? The volume of both the electron and proton and their charge states are determined by each particle's relativistic state, an expansion by $\alpha^{-1}$ for the electron and a contraction by $\alpha$ for the proton. The observed mass of each particle is determined by the spacetime curvature that localizes its energy. With their charges equal, and the same value of $G_{N}$ applicable to both particles, a similar energy configuration must exist for both. As above the electron consists of two spin- $1 / 2$ components each of $6.791 \mathrm{MeV}$, the posited unit of quantum gravity, and $\alpha^{-1} 6.7921 \mathrm{MeV}=930.76$ $\mathrm{MeV}$, close to the CODATA proton rest mass of $938.272 \mathrm{MeV}$. This suggests the proton [13] is a single spin- $1 / 2$ quantum in a highly relativistic state, with a volume contracted by $\alpha^{-1} \approx 137$ while the electron volume is expanded by 137 . The opposite and equal volume changes generate equal but opposite charge values. Consistent with current theory all but $0.724 \%$ of the proton mass is relativistic.

The empirical gravitational constant $G_{N}$ is obtained via normal matter composed mostly of protons and neutrons with the same gravitational quanta existing within all particles. If the $6.791 \mathrm{MeV}$ noted above is a gravity quantum it should relate to the proton. A particle volume change by $\alpha$ suggests a relativistic single quantum component with energy distributed nominally as $m_{c} c^{2}\left(\alpha^{-2 / 3} \cdot \alpha^{-2 / 3} \cdot \alpha^{1 / 3}\right)$. The fractional power terms presumably relate to the fractional charges assigned to quarks, but these would only be evident in the near field of protons and obviously not separable. Including

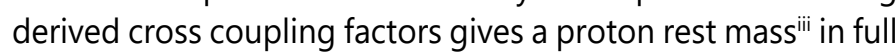
agreement with the CODATA value.

The electron volume expansion as described shows a particle in dynamic equilibrium between its mass and charge energies, $m_{e} c^{2}=\alpha \hbar c / r=e^{2} / r$. But this is not true of the proton as the apparent mass is greater than the circulating energy necessary for dynamic equilibrium. A possible solution to this problem exists if the proton energy propagates inside a closed to roidal event horizon and is therefore not in observer space, although located within it, with the circumferential energy curvature reversed and its relativistic state thereby appearing inverted on passing through the event horizon. In short, the greater mass (and proton circumferential curvature) due to an apparent volume reduction of $\alpha$ is due to a volume expansion of $\alpha^{-1}$ inside its event horizon.

\section{Understanding the MOND Constant}

Support for the posited expression for $G_{N}$ is obtained via the MOND constant at $1.24 \pm 0.14 \times 10^{-10} \mathrm{~m} / \mathrm{s}^{2}$, [14,15]. The observed flat rotation curves for stars orbiting in the outer region of galaxies are also obtained consistent with the proton being a single quantum loop.

Erroneously believing gravity radial force in the observer domain historically resulted in the gravitational attraction of a masses $M$ and $m,\left(F_{g}=G_{N} M m / R^{2}\right)$, being equated with a centripetal force $\left(F=m v^{2} / R\right)$, on a mass $m$ orbiting at velocity $V$, giving an invalid equality for an orbiting celestial body. With the advent of the GR it is now known stellar objects orbiting galaxies propagate rectilinearly and un-accelerated in curved space-time, and the assumed force equality is incorrect.

With $G_{N}=\hbar c /\left(2 m_{c} c^{2}\right)^{2}$ differing from the classical dimensions of $G_{N}$ by $c^{-4}$. To obtain an equivalent expression the invalid equality requires a dimensional adjustment; by dividing $m v^{2} / R$ by $\underline{c}^{4}$ giving

$\mathrm{G}_{\mathrm{N}} \mathrm{Mm} / \mathrm{R}^{2} \approx m v^{2} / \mathrm{R}^{4}$ 
For rotating mass systems classically constrained by a force, but not gravity, the centripetal acceleration is $v^{2} / R$. As above, this does not apply to gravitationally constrained systems where the orbiting mass is not radially accelerated. But an uninformed observer might unknowingly measure the apparent acceleration as in (8) i.e. $v^{2} / R \underline{c}^{4}$.

Orbital propagation of matter in curved space-time occurs not only in cosmological systems but also at a particle scale as described herein for both the electron and proton as electromagnetic (EM) energy localized by rectilinear propagation at a relativistic velocity in highly curved spacetime of closed geometry at the femtometer scale. For quantum energy circulating within the proton a propagation velocity $v=c\left(1-\alpha^{2 / 3}\right)^{1 / 2}$ at a posited orbit radius of $r_{p}=0.8629$ femtometers (fm) [16-19]. With these parameters, although the circulating energy is not radially accelerated, a numerical value for the apparent_classical acceleration $\mathrm{v}^{2} / \mathrm{Rc}^{4}$, can be calculated,

$$
c^{2}\left(1-\alpha^{2 / 3}\right) / r_{p} \underline{c}^{4}=1.2408 \times 10^{-10}
$$

This compares to the MOND constant as given above.

For particle stability the localizing effect maintaining the EM energy at the proton radius must have a quantum efficiency of unity, diminishing with increasing radii as $1 / r^{2}$. This attractive effect acts at the strong force scale within the particle rotating frame and as with the electron and proton is reduced to a gravitational effect in the observer domain.

\section{Flat rotation orbital stelar velocity curves}

For a large body containing $\mathrm{N}$ protons the local gravitational attraction is greater by $\mathrm{N}$ and decreases with radial distance from the body as $1 / R^{2}$. The effective quantum efficiency of the gravitational field will remain unity only out to a radius of $\mathrm{R}=\mathrm{N}^{1 / 2} \mathrm{r}_{\mathrm{p}}$. For example, with $\mathrm{N}=10^{30}$, (a mass of $1,674 \mathrm{~kg}$ ), at $r=10^{15} \mathrm{r}_{\mathrm{p}^{\prime}}$ (about $0.86 \mathrm{~m}$ ), the gravitational field of the body will have decreased by $10^{30}$ and the aggregate interaction quantum efficiency with other matter will have declined to unity, the same as at the proton radius. Beyond this critical radius the gravitating mass has insufficient gravitational field quanta to maintain the $1 / R^{2}$ decline and a field transition will occur.

At the transition radius the classically apparent angular acceleration will be as in (9), the same value as empirically determined for the MOND constant, where the rotational velocity curves of stars diverge from decreasing as $1 / R^{2}$ and become flat.

The above describes the proton as a single relativistic quantum loop, which must rotate about a tangent to tracing out a toroidal envelope. The toroidal relativistic energy configuration evident in the observer domain is posited above as $m_{q} c^{2}\left(\alpha^{-2 / 3} \cdot \alpha^{-2 / 3} \cdot \alpha^{1 / 3}\right)$, but the single loop is essentially planar and thereby the relativistic strain which forms the gravitational field is essentially one dimensional, linear and parallel to the tangent about which the energy rotates. This strain decreases as $1 / R$ for each particle and in conjunction with similar strains from a multitude of randomly oriented protons as in a macroscopic body causes the gravitational field to decrease as $1 / R^{2}$, until the transition field level is reached, after which the field decreases as $1 / R$.

At radii beyond the transition level the gravitational interaction as in (8), with $G_{N}=\hbar c /\left(m_{q} c^{2}\right)^{2}$, becomes,

$$
\left[\hbar c /\left(m_{q} \underline{c}^{2}\right)^{2}\right] M m / R=m v^{2} / R \underline{c}^{4}
$$

Which reduces to an expression independent of $R$ and $m$, i.e. $\left[\hbar c /\left(m_{q} \underline{c}^{2}\right)^{2}\right] \mathrm{Mw}=\mathrm{v}^{2} / \underline{\underline{c}}^{4}$, and re-organized shows a flat orbital velocity at all distances beyond the field transition radius.

$$
v=\left[\hbar c / m_{q}{ }^{2} M\right]^{1 / 2}
$$

The gravitational field change is not limited to orbital systems and the gravitational attraction should change, from decreasing as $1 / R^{2}$ to decreasing as $1 / R$, for static masses of any scale.

\section{Conclusion}

Several inconsistencies and erroneous assumptions are resolved enabling progress in both particle and astrophysics. Logic indicates Newton's gravity expression is fundamentally invalid with a value for $G_{N}$ deduced based on the electron as a single photon localized by rectilinear relativistic propagation in highly curved spacetime. The derived Large Number gives a direct connection between electromagnetics and General Relativity, now expanded to include the particle scale. The Planck force equality conjecture relates to the stability of elementary particles. A derived gravitational quantum energy based on the electron enables a proton rest mass energy determination within the CODATA uncertainty. The Dark Matter issue is addressed by a conjecture without requiring new particles or forces.

These realizations may enable the resumption of significant progress in Physics.

\section{References}

1. Newton I. Philososphiae Nauralis Principia. 1687.

2. Einstein A. Cosmological reflections on general relativity. Transactions of Royal Prussian Academy of Sciences (Berlin). 1917: 142.

3. Einstein Light. The Planck scale: relativity meets quantum mechanics meets gravity. The University of New South Wales.

4. Blumenthal GR, Faber SM, Primack JR, Rees MJ. Formation of galaxies and large-scale structure with cold dark matter. Nature. 1984; 311 517-525. doi: 10.1038/311517a0

5. Wolchover N. What is a Particle? Quanta Magazine. 2020.

6. Bhattacharyya G. The Hierarchy Problem and Physics Beyond the Standard Model. XXII DAE High Energy Physics Symposium. Springer Proceedings in Physics. 2018; 203. doi: 10.1007/978-3-319-73171$1 \_4$

7. Higgs P, Englert F. The Nobel Prize in Physics 2013. The Nobel Prize. 2013.

8. Dirac PAM. Cosmological Models and the Large Numbers Hypothesis Proceedings of the Royal Society of London A. 1974; 338(1615): 439446. doi: 10.1098/rspa.1974.0095

9. Cetto A, de La Pena L, Santos E. Dirac's large-number hypothesis revised. Astronomy and Astrophysics. 1986; 164(1): 1-5.

10. Ray S, Mukhopadhyay U, Ray S, Bhattacharjee A. Dirac's large number 
hypothesis: A journey from concept to implication. International Journal of Modern Physics D. 2019; 28(8): 1930014. doi: 10.1142/ S0218271819300143

11. Oakley WS. Analyzing the large number problem and Newton's $G$ via a relativistic quantum loop model of the electron. Int J Sci Rep. 2015; 1(4): 201-205. doi: 10.18203/issn.2454-2156.IntJSciRep20150671

12. Milgrom M. Does Dark matter exist? Astrophys J. 1983; 270: 365.

13. Oakley WS. Deducing the proton energy configuration. Int J Sci Rep. 2016; 2(11): 284-87. doi: 10.18203/issn.2454-2156.IntJSciRep20163968

14. McGaugh SS. A Novel Test of the Modified Newtonian Dynamics with Gas Rich Galaxies. arXiv. 2011. arXiv:1102.3913v1. 2011.
15. Oakley WS. Calculating the MOND constant and addressing flat galactic orbital star rotation velocity curves. Int J Sci Rep. 2015; 1(7): 283-286. doi: 10.18203/issn.2454-2156.IntJSciRep20151255

16. Pohl R, Antognini A, Nez F, et al. The Size of the Proton. Nature. 2010; 466: 213-216. doi: 10.1038/nature09250

17. Pohl R, Gilman R, Miller GA, Pachucki K. Muonic Hydrogen and the Proton Radius Puzzle. Annual review of Nuclear and Particle Science. 2013; 83: 175-204. doi: 10.1146/annurev-nucl-102212-170627

18. Bernauer JC, Pohl R. The Proton Radius Problem. Scientific American. 2014; 310: 32-39.

19. Oakley WS. Rethinking Physics. 3rd Edition. Red Lead Press. 2012: 64. ISBN: 978-1-4349-6734-3

'cgs is the abbreviation for cm.gm.s. units

ii A postulated energy cross axis coupling factor within the electron is given by:

$1+\alpha^{5 / 3} / 2-\left(\alpha^{5 / 3} / 2\right)^{2}+\left(\alpha^{5 / 3} / 2\right)^{3}-\left(\alpha^{5 / 3} / 2\right)^{4}+\ldots=1.0001372522$

iii A derived energy cross axis coupling factor for the proton gives the calculated proton mass energy:

$m_{p} c^{2}=\alpha^{-1} m_{q} c^{2}(1-\alpha)^{-1}\left(1+\alpha^{4 / 3} / 2\right)\left(1-\alpha^{10 / 3} / 4\right)=938.2720882 \mathrm{MeV}$, within the CODATA Standard Uncertainty.

The proton energy forms a helical toroid evident in observer space distributed nominally as $\mathrm{m}_{\mathrm{q}} \mathrm{c}^{2}\left(\alpha^{-2 / 3} \cdot \alpha^{-2 / 3} \cdot \alpha^{1 / 3}\right)$. The circumferential energy $m_{q} c^{2} \alpha^{-2 / 3}$ couples into the $z$ axis by $\alpha$ at $m_{q} c^{2} \alpha^{1 / 3}$ with a $\pi / 2$ phase shift which then couples back to the circumference by $\alpha / 2$ with another $\pi / 2$ phase shift to give the first term $\alpha^{4 / 3} / 2$. This again couples to the $z$ axis and back to the circumference reduced by $\alpha^{2} / 2$ to give the second term $\alpha^{4 / 3} / 2 \times \alpha^{2} / 2=\alpha^{10 / 3} / 4$. The initial coupling reduces the circulation by $\alpha$ which due to curvature inversion at the event horizon is evident as $(1-\alpha)^{-1}$. 